\title{
Cuatro años del Programa Nacional de Reactivovigilancia en Colombia
}

\author{
Four years of the National Reagent \\ Surveillance Program in Colombia
}

\author{
Katty A. Díaz-Roa' ${ }^{1}$, William F. Montaña-Chaparro², \\ Elkin H. Otálvaro-Cifuentes ${ }^{3}$
}

\begin{abstract}
Resumen. Introducción. El Programa Nacional de Reactivovigilancia (PNR) es un programa pionero en la región de las Américas de vigilancia poscomercialización de los reactivos de diagnóstico in vitro, en Colombia. El objetivo de este estudio fue analizar los resultados de la implementación del PNR en el periodo comprendido entre enero de 2014 y enero de 2018. Materiales y métodos. En los archivos y bases de datos del Grupo de Vigilancia Epidemiológica de la Dirección de Dispositivos y Otras Tecnologías del Invima, se realizó una búsqueda de información para caracterizar las diferentes variables del programa, las cuales se analizaron de forma descriptiva, dependiendo de su naturaleza cualitativa o cuantitativa. Resultados. Se registraron 4.191 inscripciones nuevas en la red; de ellas el 76,7\% correspondieron a entidades privadas o prestadores independientes, con cobertura aproximada del $66 \%$ de las instituciones colombianas. Se realizaron 78 capacitaciones, 93 asistencias técnicas, y 133 personas fueron certificadas de forma virtual. El $96 \%$ de los reportes fueron incidentes, 95,8\% fueron realizados por Instituciones Prestadoras de Servicios de Salud (IPS), 17,2\% debidos a falsos positivos y 44,3\% correspondieron a productos con categoría II, de acuerdo con la clasificación del Invima para el riesgo sanitario. El 74\% de los productos analizados pertenecían a la categoría II. Se reportaron 516 monitoreos internacionales. Conclusión. El PNR se constituye como el único en la región de las Américas, y después de cuatro años de iniciar su proceso de implementación, se ha logrado una cobertura importante en actores capacitados, en inscritos a la red y en el reporte de efectos indeseados.
\end{abstract}

\footnotetext{
${ }^{1}$ Bacterióloga y Laboratorista Clínica, Especialista en Gerencia de Laboratorios y Epidemiología. Profesional Especializado, Invima. Bogotá, Colombia. E-mail: kdiazr@invima.gov.co.

${ }^{2}$ Médico, Especialista en Epidemiología Clínica, MSc en Bioestadística. Profesional Especializado, Invima. Bogotá, Colombia.

${ }^{3}$ Médico, Especialista en Gerencia de Salud Pública, MSc en Seguridad Pública. Director de Dispositivos Médicos y Otras

Tecnologías, Invima. Bogotá, Colombia.

Conflicto de intereses: los autores declaran que no tienen conflicto de interés.

Medicina \& Laboratorio 2020;24:233-244. https://doi.org/10.36384/01232576.300

Recibido el 11 de septiembre de 2018; aceptado el 7 de abril de 2019. Editora Médica Colombiana S. A., 2020.
} 
Palabras clave: reactivovigilancia, vigilancia de productos, reactivos, pruebas in vitro, red.

\begin{abstract}
Introduction. The National Reagent Surveillance Program is a pioneering program in the Americas of post-marketing surveillance of in vitro diagnostic reagents in Colombia. The objective of this study was to analyze the results of the implementation of the program between January 2014 and January 2018. Methods. In the archives and databases of the Epidemiological Surveillance Group of the Office of Devices and Other Technologies of the Invima, a data search was carried out to characterize the different program variables, which were analyzed descriptively, depending on their qualitative or quantitative nature. Results. A total of 4,191 new registrations were recorded in the network; $76.7 \%$ of them from private entities or independent providers, with a $66 \%$ coverage of the Colombian institutions. There were 78 trainings, 93 technical assistance sessions, and 133 people were certified online. $96 \%$ of the reports were incidents, $95.8 \%$ were made by Health Services Provider Institutions (IPS), $17.2 \%$ due to false positives and $44.3 \%$ corresponded to products in category II, according to the Invima classification for health risk. 74\% of the products analyzed belonged to category II. 516 international monitoring reports were included. Conclusion. The PNR is a unique program in the region of the Americas, and after four years of starting its implementation process, significant coverage has been achieved with trained personnel among those enrolled in the network, and in the report of safety alerts.
\end{abstract}

Keywords: Reagent surveillance, product surveillance, reagents, in vitro testing, network.

\section{Introducción}

El Programa Nacional de Reactivovigilancia (PNR) es el programa de vigilancia poscomercialización de los reactivos de diagnóstico in vitro en Colombia. El PNR es pionero en la región de las Américas, debido a que solo Colombia cuenta con normatividad especifica frente a los reactivos de diagnóstico in vitro [1], ya que en los demás países los reactivos se encuentran inmersos dentro de la regulación de dispositivos médicos, definidos como dispositivos in vitro para uso humano; por ejemplo, lo indicado en el título 21 del Código Federal de Regulaciones de los Estados Unidos de América (CFR) en la sección 809 [2], el cual regula los requisitos para fabricantes y la distribución de los productos de diagnóstico in vitro para uso humano. Estos son considerados como dispositivos, y por lo tanto, se acogen al sistema de tecnovigilancia.

El PNR se constituye como el mecanismo de vigilancia poscomercialización de los reactivos de diagnóstico in vitro en Colombia, y tiene como propósito gestionar y evaluar sanitariamente los efectos indeseados (eventos adversos e incidentes) asociados al uso de los reactivos de diagnóstico, con el fin de tomar medidas que conduzcan a mejorar los procesos, la seguridad del paciente y de los usuarios, y de todo aquel que directa o indirectamente esté asociado con el uso de los mismos, desde una prueba de embarazo casera, la cual puede ser adquirida en una droguería, hasta pruebas de alta tecnología usadas para el diagnóstico de agentes involucrados en 
el desarrollo de enfermedades infectocontagiosas de interés en salud pública, entre otras [3-5].

Por lo expuesto, es de suma importancia realizar un análisis del programa y su proceso de implementación, el cual permita ver los resultados y el impacto en la población. En este contexto y teniendo presente que el PNR lleva cuatro años en el proceso de implementación, que está dirigido a un segmento específico de actores, y que a la fecha no se cuenta con una medición de cada una de las líneas de gestión usadas para su implementación, así como su impacto en cada departamento, esta propuesta va dirigida precisamente a realizar una evaluación de cada una de las líneas de implementación del programa, que permita estimar el impacto del mismo a nivel nacional, por grupo de actores y por departamento.

El objetivo de este trabajo es evaluar, caracterizar y analizar los resultados de la implementación del PNR en el periodo comprendido entre enero de 2014 y enero de 2018, en cuanto a las cuatro líneas de gestión: conformación de una red, capacitación y asistencia técnica a los actores del programa, gestión de los efectos indeseados y seguimiento a las alertas internacionales.

\section{Materiales y métodos}

En los archivos y bases de datos del Grupo de Vigilancia Epidemiológica de la Dirección de Dispositivos y Otras Tecnologías del Invima, se realizó una búsqueda de información relevante para caracterizar las diferentes variables del sistema de reactivovigilancia, y posteriormente se analizó cada variable de forma descriptiva, dependiendo de su naturaleza cualitativa o cuantitativa. Las variables cuantitativas se evaluaron con medidas de tendencia central y de dispersión, en tanto que las de naturaleza cualitativa se informaron con frecuencia y proporciones. En algunas ocasiones se crearon indicadores que pudieran dar una mejor claridad del impacto logrado con el programa. Adicionalmente, se analizaron los datos estratificando por año, para evaluar tendencias en el tiempo. Se utilizó la hoja de cálculo de Microsoft Excel, con sus complementos, para el análisis de la información.

\section{Resultados}

\section{Conformación de la red por grupo de actores}

Desde enero de 2014 hasta enero de 2018 se encontraron un total de 4.191 inscripciones nuevas en la red, siendo 2016 el año en que más inscripciones se realizaron $(25,29 \%)$, como se observa en la figura 1.

Con menos de 1\% (0,55\%, 23 actores) de información indeterminada, se encontró que el 76,7\% (3.213 actores) de los inscritos son entidades privadas 0 prestadores independientes; solamente un $21,94 \%$ (919 actores) pertenecía al sector público, y menos del $1 \%$ $(0,79 \%, 33$ actores) se registró como de naturaleza mixta.

En cuanto a los criterios geográficos, el $19,1 \%$ de los inscritos a la red estaban ubicados en Bogotá, particularmente, el $10,5 \%$ en Antioquia y el $70,5 \%$ restante se distribuyeron en el resto del país. En el gráfico de radar de la figura 2 se aprecia que la mayor densidad de población inscrita se encuentra en la mitad derecha del gráfico y corresponde aproximadamente al $85 \%$ de los inscritos. Las regiones del país con mayor densidad de inscritos, fueron la Antioqueña, la 


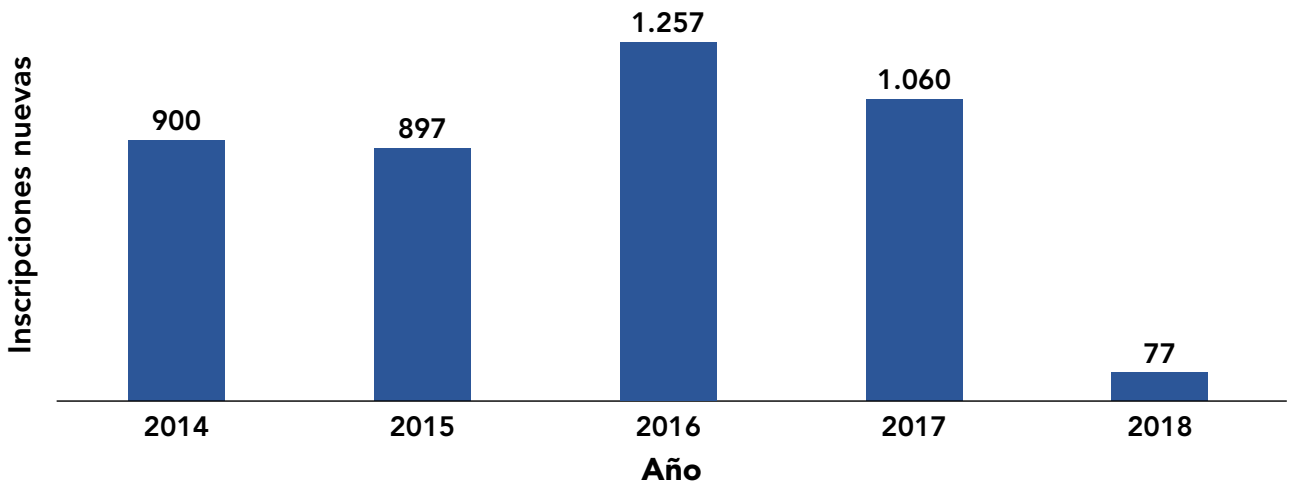

Figura 1. Frecuencia de inscripciones en la red de reactivovigilancia, por año (enero de 2014 a enero de 2018).

Cundiboyacence y la del Valle del Cauca. Todo el resto del país se encontraba con menos del $5 \%$ de los inscritos por departamento, y no se tienen registros de Vaupés ni del Amazonas (figura 3).

Al realizar una búsqueda en el Registro Especial de Prestadores de Servicios de Salud (REPS) de los actores que deberían estar inscritos en la red de reactivovigilancia, teniendo en cuenta que utilizan reactivos de diagnóstico in vitro, como laboratorios clínicos, sitios para transfusión sanguínea, laboratorios de patología, laboratorios de citología cervicouterina, laboratorios de histotecnología y bancos de sangre, se encontró una población total de 6.174 instituciones, de las que solamente el $66 \%$ estaban inscritas en el programa.

Al estratificar por departamento, se encontró que departamentos como Quindío, Risaralda, Cundinamarca, Meta, Casanare, Huila, Guainía y Vichada sobrepasaban el número total de instituciones que deberían estar inscritas, esto debido a que en muchos casos había duplicidad de inscripciones, entre otros factores. Por otra parte, departamentos como la Guajira, Mag- dalena, Bolívar y Chocó tenían menos de un $25 \%$ de las instituciones inscritas (figura 3).

\section{Cobertura de capacitación y asistencia técnica}

Para el periodo evaluado se realizaron un total de 78 eventos de capacitación; la mayor cobertura se alcanzó durante los años 2014 y 2016. En los cuatro años se tuvo un total de 4.267 personas, dentro de las que se incluyen profesionales independientes, profesionales de instituciones de servicios de salud, fabricantes e importadores. Adicionalmente, se usaron estrategias de formación virtual, a las cuales se habían inscrito 290 participantes desde el 2016 hasta la fecha, y de ellos 133 (46\%) aprobaron los cursos y fueron certificados (tabla 1).

En actividades de asistencia técnica, mediante las cuales se brinda acompañamiento y orientación a los Entes Territoriales de Salud (Secretarias de Salud) sobre la verificación de los programas de Reactivovigilancia en las IPS de su área de influencia, y sobre la implementación de medidas de 


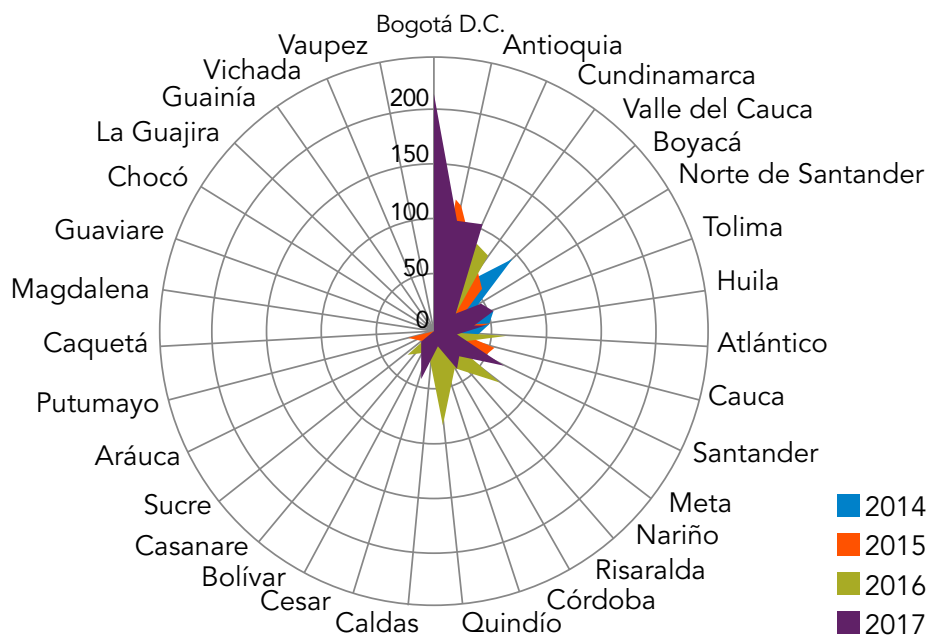

Figura 2. Densidad de inscritos a la red de reactivovigilancia estratificada por año y por departamento.
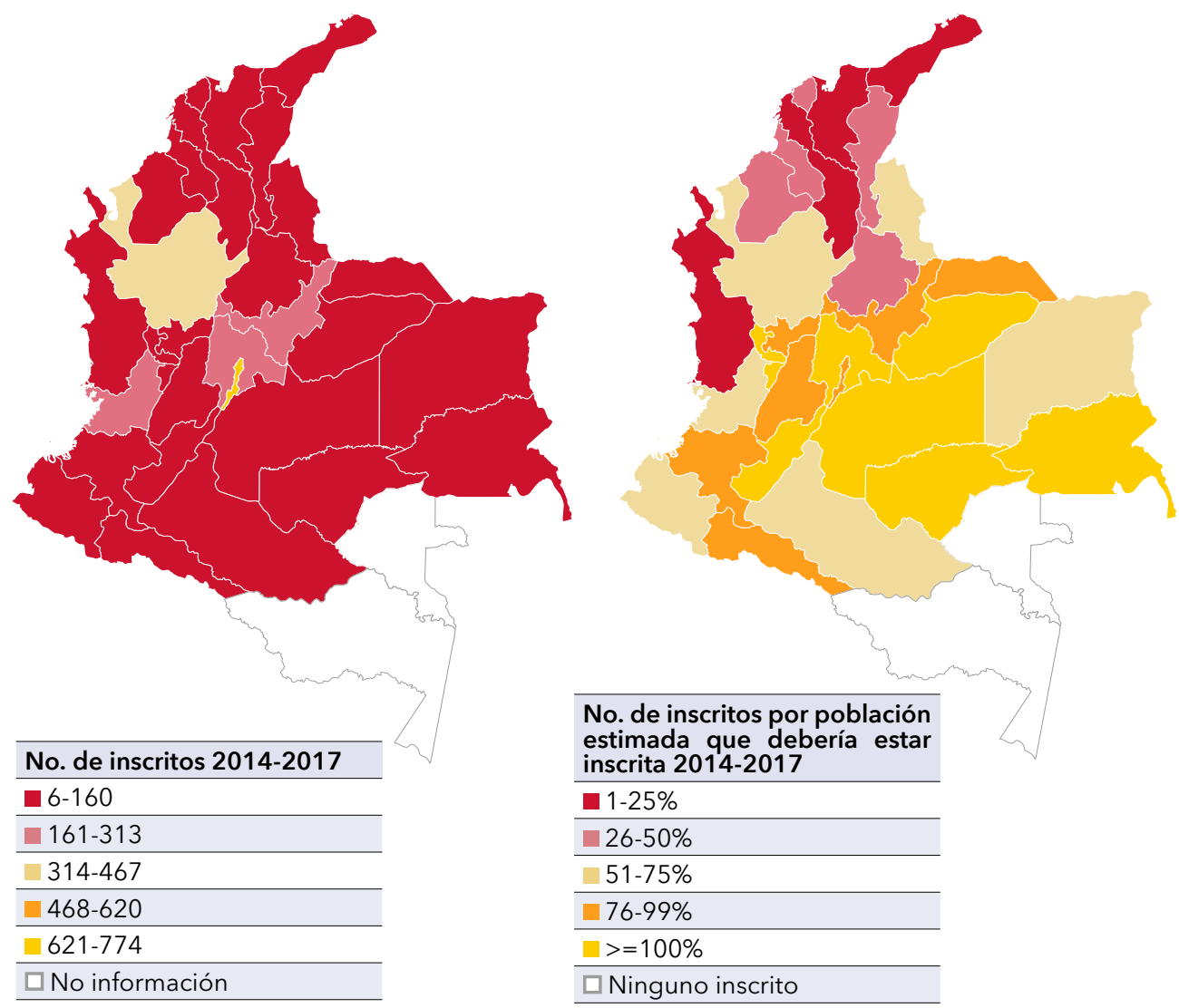

Figura 3. Mapa de frecuencias de inscritos y porcentajes de cobertura a la red por departamento desde 2014 hasta 2017. 
Tabla 1. Número de actividades y población involucrada en el Programa Nacional de Reactivovigilancia (PNR), realizado por el Invima, por año

\begin{tabular}{|c|c|c|c|c|c|c|c|c|c|c|}
\hline \multirow{2}{*}{$\begin{array}{c}\text { Tipo de } \\
\text { actividades }\end{array}$} & \multicolumn{2}{|c|}{2014} & \multicolumn{2}{|c|}{2015} & \multicolumn{2}{|c|}{2016} & \multicolumn{2}{|c|}{2017} & \multicolumn{2}{|c|}{ Total } \\
\hline & $\mathbf{n}$ & $\%$ & $\mathbf{n}$ & $\%$ & $\mathbf{n}$ & $\%$ & $\mathbf{n}$ & $\%$ & $\mathbf{n}$ & $\%$ \\
\hline \multirow{2}{*}{$\begin{array}{l}\text { Capacitaciones } \\
\text { Participantes }\end{array}$} & 19 & 24,4 & 13 & 16,7 & 28 & 35,9 & 18 & 23,1 & 78 & 100 \\
\hline & 1.401 & 32,8 & 599 & 14,0 & 1.526 & 35,8 & 741 & 17,4 & 4.267 & 100 \\
\hline $\begin{array}{l}\text { Participación } \\
\text { virtual }\end{array}$ & - & - & - & - & 140 & 48,3 & 150 & 51,7 & 290 & 100 \\
\hline Certificados & - & - & - & - & 64 & 48,1 & 69 & 51,9 & 133 & 100 \\
\hline $\begin{array}{l}\text { Asistencias } \\
\text { técnicas }\end{array}$ & 13 & 14,0 & 16 & 17,2 & 34 & 36,6 & 30 & 32,3 & 93 & 100 \\
\hline Participantes & 149 & 17,2 & 187 & 21,6 & 280 & 32,3 & 250 & 28,9 & 866 & 100 \\
\hline
\end{tabular}

No se realizaron actividades en enero de 2018

control y seguimiento de los mismos, así como acompañamiento a los prestadores respecto a la implementación de AMFE (Análisis de Modos de Fallo y sus Efectos) como Sistema de Gestión de Riesgo Clínico para la prevención de efectos indeseados asociados a Reactivos de Diagnóstico in vitro, la mayor cobertura en eventos y participantes se obtuvo durante los años 2016 (34 eventos de 93) y 2017 (30 eventos de 93).

Al hacerse el análisis de cuántas capacitaciones se realizaron durante el periodo 2014 a 2017, se observó que la gran mayoría fue realizada en el año 2016, y Bogotá fue la ciudad en donde se realizó la mayor cantidad de ellas.

\section{Efectos indeseados reportados al PNR}

Se observó un aumento constante anual de los reportes al Programa de Reactivovigilancia, pasando de 55 reportes en 2014, a 254 en 2017 (tabla 2). El número de reportes tuvo una tendencia lineal, en donde por cada año hubo un aumento promedio de 62 reportes, con lo que se esperaría para el 2018, con la misma tendencia, unos 316 reportes, aproximadamente (figura 4).

Al evaluar el tipo de reporte, el $4 \%$ de ellos fue considerado como un evento adverso y el $96 \%$ restante fue clasificado como incidente. Con respecto a la institución reportante, la gran mayoría

Tabla 2. Clasificación del evento reportado estratificado por año

\begin{tabular}{lrcccc}
\hline Año & $\begin{array}{l}\text { Evento } \\
\text { adverso }\end{array}$ & $\begin{array}{l}\text { Frecuencia } \\
\text { incidente }\end{array}$ & Total reportes & $\begin{array}{l}\text { Frecuencia } \\
\text { relativa (\%) }\end{array}$ & $\begin{array}{l}\text { Frecuencia } \\
\text { absoluta (\%) }\end{array}$ \\
\hline 2014 & 7 & 48 & 55 & 8,44 & 8,44 \\
\hline 2015 & 10 & 128 & 138 & 21,17 & 29,60 \\
\hline 2016 & 7 & 164 & 171 & 26,23 & 55,83 \\
\hline 2017 & 4 & 250 & 254 & 38,96 & 94,79 \\
\hline 2018 & 0 & 34 & 34 & 5,21 & 100,00 \\
\hline Total & 28 & 624 & 652 & 100,00 & \\
\hline
\end{tabular}




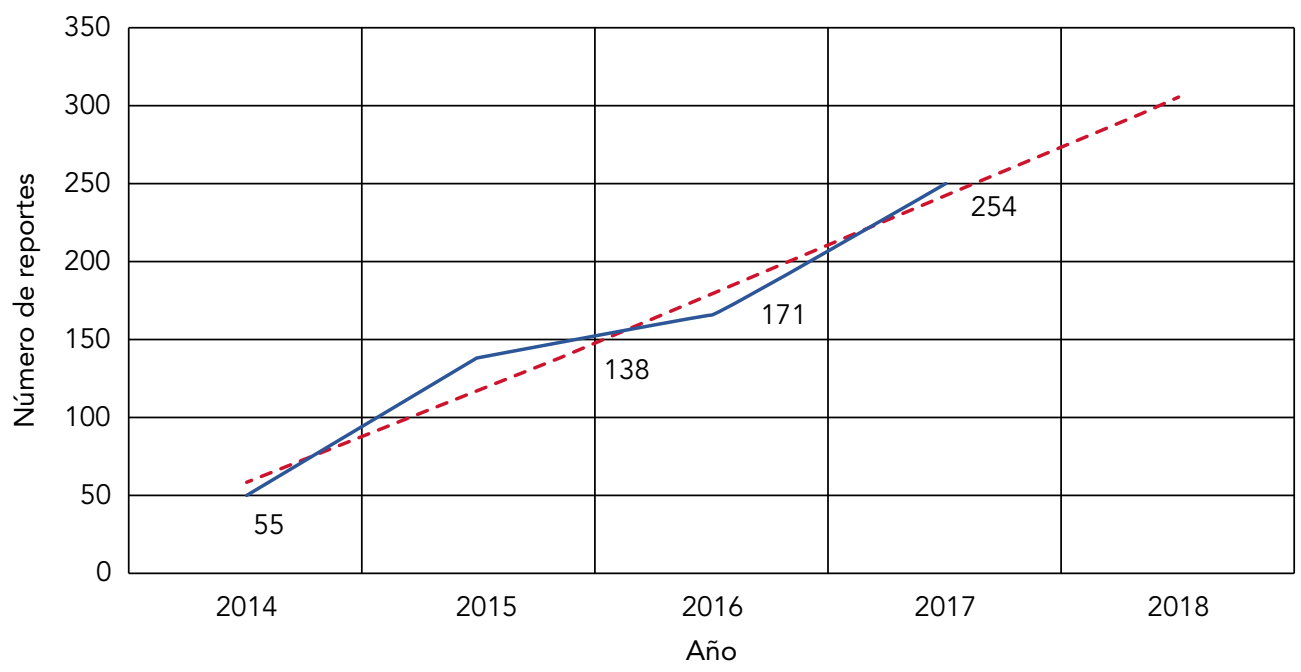

Figura 4. Número anual de reportes totales y línea de tendencia al año 2018.

de reportes fue realizada por una IPS (95,85\%), y en mínima cantidad por el propio importador o por Secretarías Regionales (3,23\% y 0,92\%, respectivamente). Los reportes se dieron principalmente de productos de categoría II $(44,31 \%)$ y categoría III $(40,46 \%)$, y en muy baja proporción en los de categoría I (14,15\%), clasificados de acuerdo con el riesgo sanitario [6].

Al evaluar la causa del reporte, se encontró que los falsos positivos, con un $17,18 \%$, fueron los más frecuentes, seguidos de resultados falsos negativos de la prueba $(14,11 \%)$. También es de resaltar que una de las principales causas encontradas en el análisis de los reportes fue el error en el uso de los productos (5,21\%) (figura 5).

Al comparar por departamento la población inscrita existente para generar un reporte al Programa, se encontró que a nivel nacional habían 6 instituciones inscritas por reporte durante el periodo, con el mejor índice en Valle del Cauca y Bogotá, con 3 instituciones inscritas por reporte; el indicador más bajo se en- contró en Quindío con 107 instituciones inscritas y un solo reporte (tabla 3). Los 87 inscritos de Guainía, Guaviare, Putumayo, San Andrés/Providencia, Vaupés y Vichada, en los más de cuatro años, no realizaron ningún reporte.

\section{Alertas y retiros de productos, e informes de seguridad}

Durante el periodo entre enero de 2014 a enero de 2018 se monitorearon 516 reportes de productos comercializados en Colombia que tenían alertas, informes de seguridad o retiros en bases de datos internacionales (tabla 4). En el año 2016 se reportó la mayor cantidad de casos del periodo $(n=179), y$ en 2014 la menor $(n=75)$. En promedio, 126,5 reportes de productos fueron gestionados por año en estos últimos cinco años. No se encuentra aparentemente ninguna línea de tendencia definida. Es de resaltar que durante el año 2018 , solamente se tomaron los reportes monitoreados en el mes de enero, por lo que solo 10 fueron evaluados en la base de datos. 


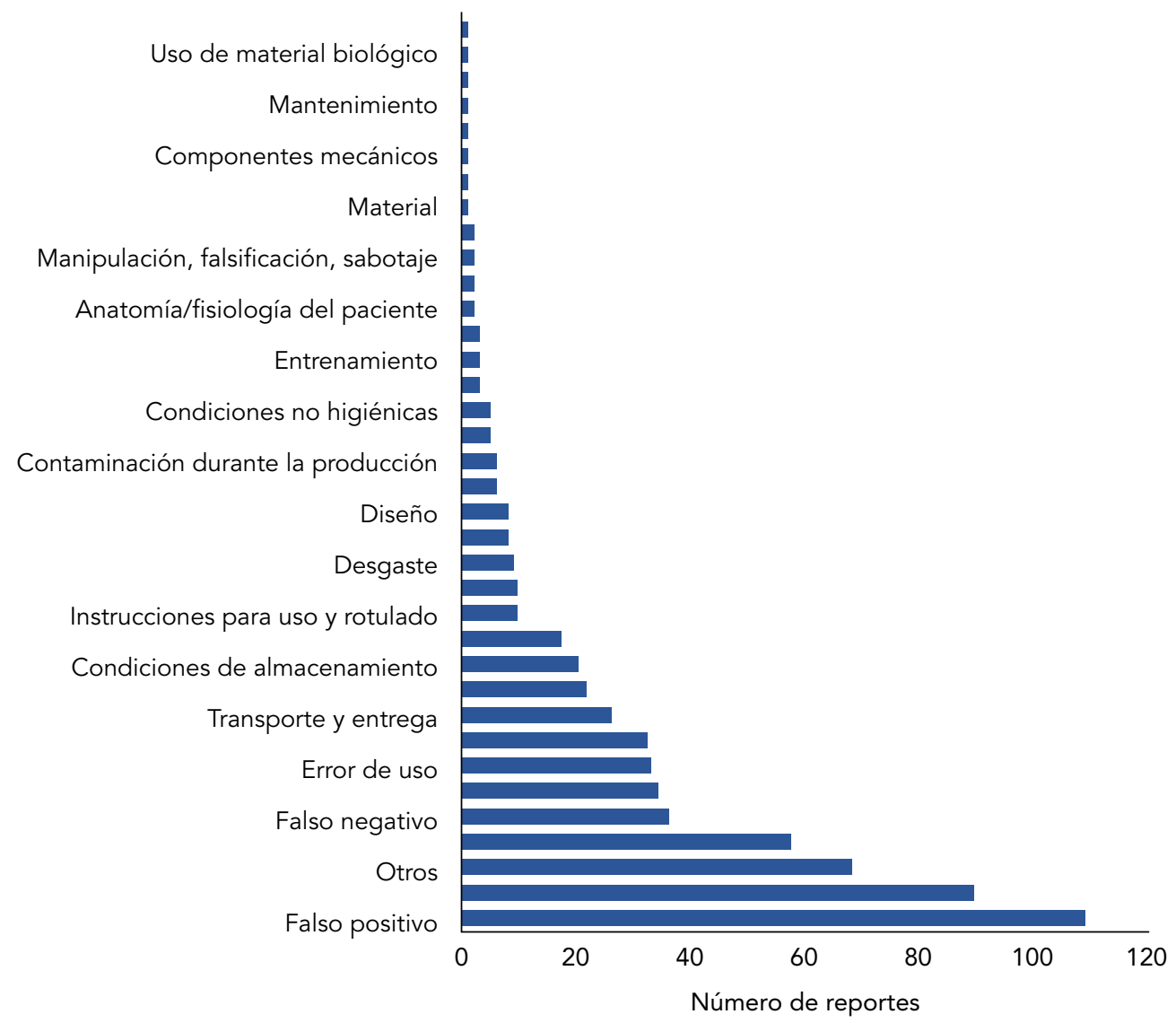

Figura 5. Frecuencia de reportes según la descripción de la causa.

Del total de reportes monitoreados, el $47 \%$ correspondió a informes de seguridad, el $44 \%$ a recogidas del mercado, y un $5 \%$ a alertas. Al evaluar por año, la menor cantidad de monitoreos se realizaron sobre alertas, en tanto que los retiros del producto y los informes de seguridad, se alternaron en frecuencia, anualmente.

En cuanto al seguimiento a las alertas internacionales, durante el periodo entre enero 2014 a enero 2018, se monitorearon 516 casos de productos comercializados en Colombia. De estos, 395 fueron reportes únicos de productos, pero existiendo en ocasiones hasta 6 diferentes reportes para un solo producto. La principal agencia internacional que aportó la mayoría de reportes fue la ANSM (del francés, Agence Nationale de Sécurité du Médicament) de Francia (39,73\%), seguida de la MHRA (del inglés, Medicines and Healthcare Products Regulatory Agency) del Reino Unido (15,12\%) [3].

Cuando se evaluó la categoría del reactivo que fue monitoreado durante el periodo de evaluación, se encontró que la mayoría perteneció a la categoría II (74\%), en varios subgrupos, y en una menor cantidad a la categoría III (17\%) y a la categoría I (8\%). Se estimó una pérdida de información que no superó el $3,5 \%$ de los datos. 


\begin{tabular}{|c|c|c|c|c|}
\hline Departamento & Reportes & Inscritos & $\begin{array}{l}\text { Indicador } \\
\text { de reporte* }\end{array}$ & $\begin{array}{l}\text { Tasa anual } \\
\text { del indicador }\end{array}$ \\
\hline Valle del Cauca & 80 & 250 & 3,1 & 0,62 \\
\hline Bogotá & 246 & 774 & 3,1 & 0,62 \\
\hline Antioquia & 132 & 425 & 3,2 & 0,64 \\
\hline Santander & 29 & 132 & 4,6 & 0,92 \\
\hline Casanare & 11 & 69 & 6,3 & 1,26 \\
\hline Córdoba & 13 & 111 & 8,5 & 1,7 \\
\hline Cauca & 16 & 139 & 8,7 & 1,74 \\
\hline Magdalena & 4 & 39 & 9,8 & 1,96 \\
\hline Atlántico & 14 & 150 & 10,7 & 2,14 \\
\hline Boyacá & 19 & 209 & 11,0 & 2,2 \\
\hline Risaralda & 11 & 127 & 11,5 & 2,3 \\
\hline Sucre & 5 & 63 & 12,6 & 2,52 \\
\hline Chocó & 1 & 13 & 13,0 & 2,6 \\
\hline La Guajira & 1 & 13 & 13,0 & 2,6 \\
\hline Huila & 10 & 151 & 15,1 & 3,02 \\
\hline Cesar & 5 & 86 & 17,2 & 3,44 \\
\hline Tolima & 9 & 157 & 17,4 & 3,48 \\
\hline Caldas & 6 & 105 & 17,5 & 3,5 \\
\hline Meta & 7 & 129 & 18,4 & 3,68 \\
\hline Cundinamarca & 14 & 269 & 19,2 & 3,84 \\
\hline Nariño & 5 & 128 & 25,6 & 5,12 \\
\hline Bolívar & 3 & 78 & 26,0 & 5,2 \\
\hline Norte de Santander & 5 & 159 & 31,8 & 6,36 \\
\hline Caquetá & 1 & 39 & 39,0 & 7,8 \\
\hline Arauca & 1 & 50 & 50,0 & 10 \\
\hline Quindío & 1 & 107 & 107,0 & 21,4 \\
\hline Total & 649 & 3972 & 6,1 & 1,22 \\
\hline
\end{tabular}

*Inscritos/Reportes 


\begin{tabular}{|c|c|c|c|c|c|c|c|c|c|c|c|c|}
\hline \multirow[b]{2}{*}{ Clase de reporte } & \multicolumn{2}{|c|}{2014} & \multicolumn{2}{|c|}{2015} & \multicolumn{2}{|c|}{2016} & \multicolumn{2}{|c|}{2017} & \multicolumn{2}{|c|}{2018} & \multicolumn{2}{|c|}{ Total } \\
\hline & $\mathbf{n}$ & $\%$ & $\mathbf{n}$ & $\%$ & $\mathbf{n}$ & $\%$ & $\mathbf{n}$ & $\%$ & $\mathbf{n}$ & $\%$ & $\mathbf{n}$ & $\%$ \\
\hline Alerta & 0 & 0 & 2 & 2 & 14 & 8 & 8 & 7 & 1 & 10 & 25 & 5 \\
\hline $\begin{array}{l}\text { Informe de } \\
\text { seguridad }\end{array}$ & 10 & 13 & 79 & 60 & 89 & 50 & 62 & 52 & 5 & 50 & 245 & 47 \\
\hline $\begin{array}{l}\text { Recogida } \\
\text { del mercado }\end{array}$ & 64 & 85 & 50 & 38 & 76 & 42 & 35 & 29 & 4 & 40 & 229 & 44 \\
\hline Indeterminado* & 1 & 1 & 1 & 1 & 0 & 0 & 15 & 13 & 0 & 0 & 17 & 3 \\
\hline Total & 75 & 100 & 132 & 100 & 179 & 100 & 120 & 100 & 10 & 100 & 516 & 100 \\
\hline
\end{tabular}

*En 17 casos no fue posible determinar la clasificación del reporte

\section{Discusión}

El PNR se constituye como el único en la región de las Américas, y luego de un tiempo relativamente corto de haber iniciado su proceso de implementación, ha conseguido una cobertura importante de inscritos a la red, actores capacitados, y en el reporte de efectos indeseados.

Para el caso específico de las inscripciones a la red, se observan departamentos que están en el rango entre $1 \%$ y $25 \%$ de cobertura de inscritos; no obstante, al verificar la actualidad de dichas zonas se encuentra que son departamentos que no cuentan con referente estable del Programa, tienen una alta rotación de personal o no cuentan con sistemas robustos que permitan la adherencia a los programas. Adicionalmente, son zonas con muy difícil acceso terrestre y redes altamente inestables.

En la Red Nacional de Reactivovigilancia, la tendencia para el año 2018 no indicaba que el número de inscritos aumentaría en mayor medida de lo que se venía presentando en los cuatro años previos. Es de resaltar que el número total de inscritos incluyó prestadores independientes. El no alcanzar un $100 \%$ de cobertura puede radicar en un control insuficiente en algunas regiones del país por la ausencia de referentes en cada departamento, o por un subregistro de las entidades habilitadas de las que se esperaba que estuvieran inscritas al Programa. En un esfuerzo por alcanzar alta cobertura y eficiencia, y contando con presupuestos ajustados, la red de reactivovigilancia, mediante estrategias de capacitación y asistencia técnica, ha logrado evolucionar a plataformas virtuales de tipo "e-learning", mediante las cuales se ha impactado un mayor número de actores, tanto nacionales como internacionales.

Respecto a las capacitaciones y asistencias técnicas, se ha logrado una cobertura importante a nivel nacional e internacional; no obstante, con la inclusión de las nuevas tecnologías de formación virtual, se espera lograr una cobertura mayor, con una retención en los procesos que supere el $80 \%$. Así mismo, es importante fortalecer mediante la implementación de Sistemas de Gestión de Riesgo Clínico, la 
vigilancia proactiva, de tal forma que se estructure una red que le permita a las instituciones mejorar sus procesos, compartir información y realizar una vigilancia transversal a los reactivos de diagnóstico in vitro.

Al cruzar la información del número de inscritos a la red por departamento con el número de capacitaciones, se pudo evidenciar que departamentos como Boyacá, Cundinamarca y Risaralda tuvieron una mayor cantidad de inscritos en proporción con la baja cantidad de capacitaciones realizadas en los cuatro años. El caso contrario sucedió con el Magdalena, Chocó y la Guajira, que tuvieron una proporción de capacitaciones mayor que el número de inscritos a la red. Aunque el coeficiente de correlación de Pearson muestra una posible correlación lineal entre los inscritos y las capacitaciones, $\left(r^{2}=0,74\right)$, lo cual indicaría que, a mayor cantidad de capacitaciones, se incrementan los inscritos, no puede aseverarse esto, pues la inscripción pudo corresponder a otros mecanismos que aquí no fueron evaluados. Los reportes no muestran correlación alguna con la cantidad de capacitaciones realizadas en el periodo por cada departamento $\left(r^{2}=0,012\right)$. Esto es algo difícil de interpretar, al igual que estimar la influencia que tuvieron las capacitaciones en la cantidad de inscritos en la red; sin embargo, el año en que se realizaron el mayor número de capacitaciones fue el año en que hubo el mayor número de inscritos (año 2016, con 28 capacitaciones y 1.180 inscritos). Para el caso de si los reportes fueron influenciados por la cantidad de capacitaciones en cada departamento, se puede apreciar que tampoco es clara esta relación; los departamentos con centros poblacionales más desarrollados, tuvieron baja relación de capacitaciones y reportes; es decir, se realizaron capacitaciones, pero los reportes fueron muy pocos. Es de resaltar, que la relación entre reportes y cantidad de inscritos por departamento, sí tuvo una correlación directa, lo cual era lo esperado.

Se estima que el aumento constante del número de reportes por año tenderá a estabilizarse en los próximos tres años, y que las inscripciones no aumenten más de lo que aumente la cantidad de instituciones obligadas a inscribirse, esto si continúan las mismas políticas y prácticas en el Programa de Reactivovigilancia. Es decir, los esfuerzos deberán concentrarse sobre los departamentos que aún no tienen una cultura del reporte, y en que se inscriban las instituciones que por ley deberían estarlo, esfuerzos que deben coordinarse con los referentes del Programa en cada departamento.

Como parte del inicio de un programa de vigilancia sanitaria y al ser un programa pionero en la región relativamente nuevo, los reportes nacionales deben estar complementados con referentes extranjeros con productos comercializados dentro del país. Es por esto que la labor de monitoreo que se realiza es crucial para una red joven, ya que por la baja cantidad de reportes, debe tratar de anticiparse a los riesgos asociados con el uso de los reactivos.

Finalmente, con relación a las alertas, los retiros de productos y los informes de seguridad, estos son a demanda; aún no se ha logrado establecer una tendencia que permita proyectar su movimiento. Sin embargo, son actividades que deben fortalecerse, en cuanto a los mecanismos de comunicación del riesgo.

\section{Fuente de financiación}

Invima participó en la decisión de enviar el artículo para su publicación y en los gastos propios de la contratación de los autores. 


\section{Referencias}

1. Instituto Nacional de Vigilancia de Medicamentos y Alimentos (INVIMA). Resolución 2013038979 de 2013. «Por medio de la cual se implementa el Programa Nacional de Reactivovigilancia». Diario Oficial No. 49.048 de 29 de enero de 2014. Acceso 14 de julio de 2018. Disponible en https://www.icbf.gov.co/cargues/avance/ docs/resolucion_invima_38979_2013.htm.

2. U.S. Food and Drug Administration. Code of Federal Regulations, Title 21. Maryland: U.S. Department of Health and Human Services; 2019. Volume 8: Part 809. Acceso 23 de julio de 2018. Disponible en https://www.accessdata. fda.gov/scripts/cdrh/cfdocs/cfCFR/CFRSearch. cfm?CFRPart=809.

3. Montaña-Chaparro WF, Díaz-Roa KA, Otalvaro-Cifuentes EH. Estudio descriptivo del Programa Nacional de Reactivovigilancia. Bogotá D. C.: INVIMA; 2018. p. 33. Acceso 3 de septiembre de 2018. Disponible en https://www. invima.gov.co/documents/20143/442961/ ESTUDIO-DESCRIPTIVO-PROGRAMA-DEREACTIVOVIGILANCIA-EN-COLOMBIA-SEPTIEMBRE-2018.pdf/f48dc56f-4624-82f0-d35be4b935fc0d66?t=1560457322234.
4. Ministerio de la Protección Social. Decreto número 3770 de 2004. Por el cual se reglamentan el régimen de registros sanitarios y la vigilancia sanitaria de los reactivos de diagnóstico in vitro para exámenes de especímenes de origen humano. Bogotá D. C., 12 de noviembre del 2004. Acceso 5 de agosto de 2018. Disponible en https://www.minsalud.gov.co/Normatividad_Nuevo/DECRETO\%203770\%20DE\%20 2004.PDF.

5. Ministerio de Salud y Protección Social. Resolución número 1229 de 2013. Por la cual se establece el modelo de inspección, vigilancia y control sanitario para los productos de uso y consumo humano. Bogotá D. C. 23 de abril del 2013. Acceso 18 de junio de 2018. https://www. minsalud.gov.co/sites/rid/Lists/BibliotecaDigital/RIDE/DE/DIJ/resolucion-1229-de-2013.pdf.

6. Ministerio de Salud y Protección Social. Instituto Nacional de Vigilancia de Medicamentos y Alimentos (INVIMA). ABC de Reactivovigilancia. Bogotá D. C: Imprenta Nacional de Colombia; 2014. p. 39. Acceso 1 de julio del 2018. Disponible en https:// es.scribd.com/document/375066271/ABCReactivovigilancia. 